\title{
Facile and Efficient Method for Synthesis of Benzimidazole Derivatives Catalyzed by Zinc Triflate
}

\author{
Ramineni Srinivasulu' ${ }^{1}$, Kannasani Ravi Kumar ${ }^{2}$, Peruri Veera Venkata Satyanarayana ${ }^{3}$ \\ ${ }^{1}$ Chalapathi Institute of Engineering and Technology, Department of chemistry, Guntur, India \\ ${ }^{2}$ RA Chem Pharma Limited, R\&D Division, Prasanth Nagar, Hyderabad, India \\ ${ }^{3}$ Department of Chemistry, Acharya Nagarjuna University, Guntur, India \\ Email: ramineni29@yahoo.com
}

Received December 4, 2013; revised January 4, 2014; accepted January 11, 2014

Copyright (c) 2014 Ramineni Srinivasulu et al. This is an open access article distributed under the Creative Commons Attribution License, which permits unrestricted use, distribution, and reproduction in any medium, provided the original work is properly cited. In accordance of the Creative Commons Attribution License all Copyrights (C) 2014 are reserved for SCIRP and the owner of the intellectual property Ramineni Srinivasulu et al. All Copyright @ 2014 are guarded by law and by SCIRP as a guardian.

\begin{abstract}
We report the synthesis of benzimidazole derivatives using zinc triflate as an efficient catalyst. One-pot synthesis of 2-substituted benzimidazole derivatives from o-phynelyenediamine and substituted aldehydes were developed under zinc triflate in ethanol solvent at reflux temperature.
\end{abstract}

\section{KEYWORDS}

\section{Benzimidazole Derivatives; O-Phynelyenediamine; Substituted Aldehydes; Zinc Triflate}

\section{Introduction}

Benzimidazole derivatives have received much interest in the field of medicinal chemistry [1,2]. Benzimidazole group of substances has found practical applications in a number of fields. Recently the interest in benzimidazole chemistry has been revived by the discovery that the 5,6dimethyl benzimidazole moiety is part of the chemical structure of vitamin B12 [3]. Substituted Benzimidazoles display a broad spectrum of potential pharmacological activities and are present in a number of pharmacologically active molecules such as albendazole/mebendazole/ thiabendazole (antihelmentic), omeprazole (anti-ulcer), etc. (Figure 1). Considerable interest has been focused on the benzimidazole structure. The discovery of this class of drugs provides an outstanding case history of modern drug development and also points out the unpredictability of pharmacological activity from structural modification of a prototype drug molecule. It is having a variety of medicinal applications. Benzimidazole derivatives carrying different substituent's in the benzimidazole structure were associated with a wide range of biological activities including anticancer, antiviral, antibacterial, antifungal, antihelmentic, anti-inflammatory, antihistaminic, proton pump inhibitor, antioxidant, antihypertensive and anticoagulant activities. Their derivatives were also found to exhibit cytotoxic activity. Substituted benzimidazole derivatives are evaluated by their ability to inhibit gastric $\mathrm{H}^{+} / \mathrm{K}^{+}$ATPase and by blocking the gastric acid secretion [4]. Recently, benzimidazoles have also been used as ligands for asymmetric catalysis [5].

Many methods have been reported for the synthesis of these benzimidazole derivatives. The condensation of 1,2-phenylenediamines with carboxylic acids or their derivatives is a common method, but it needs harsh conditions like polyphosphoric acid [6] at $170^{\circ} \mathrm{C}-180^{\circ} \mathrm{C}$. Another alternative approach is the condensation of aldehyde with 1,2-phenylenediamine in presence of different catalysts like Indion 190 resin [7], $\mathrm{BF}_{3} . \mathrm{OEt}_{2}$ [8], Ceric ammonium nitrate [9], iodine, [10] Silica sulfuric acid [11], $\mathrm{In}(\mathrm{OTf})_{3}$ [12], $\mathrm{SiO}_{2} / \mathrm{ZnCl}_{2}$ [13], silica supported sodium hydrogen sulphate [14], PEG [15], $\mathrm{H}_{2} \mathrm{O}_{2} /$ $\mathrm{Fe}\left(\mathrm{NO}_{3}\right)_{3}$ [16]. In recent years, Solvent-free synthesis of benzimidazoles under microwave irradiation using $\mathrm{Yb}(\mathrm{OTf})_{3}$ [17], KSF clay [18], metal halide supported alumina [19] and solid support [20,21] has been reported. However, many of these methods suffer from one or more drawbacks such as requirement of strong acidic conditions, long reaction times, low yields, tedious workup procedures, requirement of excess amounts of reagents, and use of toxic reagents, catalysts or solvents. 


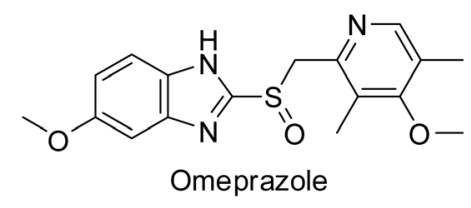<smiles>Cc1c(OCC(F)(F)F)ccnc1CS(=O)c1nc2ccccc2[nH]1</smiles><smiles>COc1ccnc(CS(=O)c2nc3cc(OC(F)F)ccc3[nH]2)c1OC(=O)[O-]</smiles><smiles>COCCCOc1c(C)cnc(CS(=O)c2nc3ccccc3[nH]2)c1C</smiles><smiles>[R6][R]O[Na]</smiles>

Figure 1. Established antiulcer agents in clinical practice.

Therefore, there is a strong demand for a highly efficient and environmentally benign method for the synthesis of these heterocycles.

As part of our research program in developing various synthetic methodologies, we report the synthesis of benzimidazoles using zinc triflate as an efficient catalyst (Scheme 1). The catalyst is known as an efficient catalyst in the literature for various organic transformations [22-26].

\section{Results and Discussions}

In order to establish the optimum reaction condition for this reaction, different solvents and various mole ratios of zinc triflate were examined. In our preliminarily investigation was carried out on the model reaction of o-phenylenediamine and 4-methoxy benzaldehyde. As shown in Table 1, different solvents can result in different yields. It was found that ethanol is the best solvent for condensation reaction, with its fast conversion, high yield and low toxicity. Zinc triflate was added in various mole ratios in ethanol at reflux. As shown in Table 2. The best yields were obtained with $10 \mathrm{~mol} \%$ of zinc triflate. The electronic effects of the different substituted aldehydes have been investigated in Table 3 and it was observed that aldehydes bearing both electron donating and electron with drawing substituents gave the desired benzimidazoles in good yields. Products were confirmed by comparing with authentic sample $\left({ }^{1} \mathrm{H}\right.$ NMR, MR and Mass).

\section{Conclusions}

In conclusion, Zinc triflate was found to be an efficient catalyst for the formation of benzimidazole from aldehydes and $o$-phenylenediamine. The use of this inexpensive and easily available catalyst makes this protocol practical, environment friendly and economically attrac-

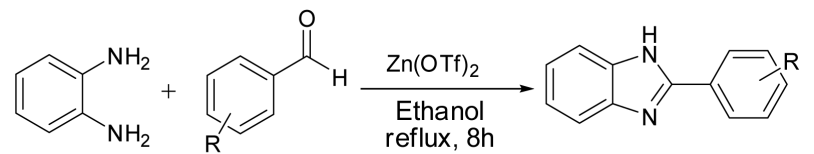

Scheme 1. Synthesis of Benzimidazole derivatives catalyzed by zinc triflate.

Table 1. Effect of Solvent in the synthesis of 2-(4-Methoxyphenyl) benzimidazole.

\begin{tabular}{ccccc}
\hline Entry & Solvent & Temperature $\left({ }^{\circ} \mathrm{C}\right)$ & Time (hr) & Yield $(\%)^{\mathrm{a}}$ \\
\hline 1 & $\mathrm{CH}_{2} \mathrm{Cl}_{2}$ & 40 & 12 & 58 \\
2 & $\mathrm{CH}_{3} \mathrm{OH}$ & 65 & 10 & 70 \\
3 & $\mathrm{CH}_{3} \mathrm{CH}_{2} \mathrm{OH}$ & 80 & 8 & 95 \\
4 & $\mathrm{THF}$ & 68 & 12 & 62 \\
5 & $\mathrm{CH}_{3} \mathrm{CN}$ & 85 & 10 & 72 \\
\hline
\end{tabular}

${ }^{\mathrm{a}}$ All are isolated yields.

Table 2. various mole ratios of zinc triflate for the synthesis of 2-(4-Methoxyphenyl) benzimidazole.

\begin{tabular}{cccc}
\hline Entry & Zinc triflate (mol\%) & Time (hr) & Yield (\%) $^{\mathrm{a}}$ \\
\hline 1 & 0 & 12 & 18 \\
2 & 5 & 8 & 68 \\
3 & 10 & 8 & 95 \\
4 & 15 & 8 & 95 \\
5 & 20 & 8 & 94 \\
\hline
\end{tabular}

${ }^{\mathrm{a}}$ All are isolated yields.

tive. The simple work-up procedure, high yields of products and nontoxic nature of the catalyst are other advantages of the present method.

\subsection{Experimental}

All ${ }^{1} \mathrm{H}$ NMR spectra were recorded on $400 \mathrm{MHz}$ Varian FT-NMR spectrometers. All chemical shifts are given as $\delta$ 
Table 3. synthesis of 2-substituted benzimidazoles from $O$-Phenylenediamine and aldehydes ${ }^{\mathrm{a}}$.

Entry

aldehyde

Benzimidazole

Yield $^{\mathrm{b}}$

1<smiles>O=Cc1ccccc1</smiles>

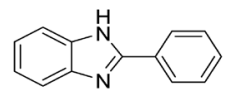

94

2<smiles>O=Cc1ccccc1Cl</smiles><smiles>Clc1ccccc1-c1nc2ccccc2[nH]1</smiles>

3<smiles>O=Cc1cccc(Cl)c1</smiles>

4<smiles>O=Cc1ccc(Cl)cc1</smiles><smiles>Clc1ccc(-c2nc3ccccc3[nH]2)cc1</smiles>

89

5<smiles>Cc1ccccc1C=O</smiles>

6<smiles>Cc1ccc(C=O)cc1</smiles>

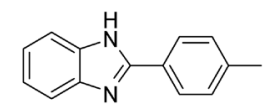

90

7<smiles>COc1ccccc1C=O</smiles><smiles>COc1ccccc1-c1nc2ccccc2[nH]1</smiles>

90

8<smiles>COc1ccc(C=O)cc1</smiles><smiles>COc1ccc(-c2nc3ccccc3[nH]2)cc1</smiles>
95<smiles>O=Cc1cccc([N+](=O)[O-])c1</smiles>

10

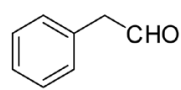

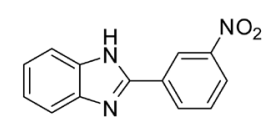

81

95

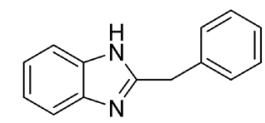

3.2. Zinc Triflate Catalyzed Synthesis of 2-Substituted Benzimidazole Derivatives from Aldehydes

A mixture of $o$-phenylenediamine ( $1 \mathrm{mmol})$, benzaldehyde $(1.0 \mathrm{mmol})$ and $\mathrm{Zn}(\mathrm{OTf})_{2}(10 \mathrm{~mol} \%)$ in Ethanol (5 $\mathrm{ml}$ ) was placed in a $50 \mathrm{ml}$ round bottom flask and stirred at reflux for $8 \mathrm{~h}$. The progress of the reaction was monitored by TLC Hexane: EtOAc (8:2) after completion of the reaction, the reaction mixture was cooled and treated by dilution with EtOAc $(20 \mathrm{~mL})$. Total organic layer was washed with water, brine solution and dried over $\mathrm{Na}_{2} \mathrm{SO}_{4}$ and evaporated under vacuum. Obtained crude residue was purified by column chromatography to give 2-substituted benzimidazoles.

2-Phenylbenzimidazole [27]: Off white solid; m.p: $289^{\circ} \mathrm{C}-291^{\circ} \mathrm{C} ;{ }^{1} \mathrm{H}$ NMR (DMSO-d 6 ): $\delta 13.02$ (br s, $1 \mathrm{H}$ ), 8.20 (d, $J=7.6 \mathrm{~Hz}, 2 \mathrm{H}), 7.67-7.65$ (m, 1H), $7.56-7.49$ (m, 4H), 7.22 - 7.18 (m, 2H); (LC-MS) m/z: 195.08 [M + $\mathrm{H}]^{+}$; IR (KBr, $\left.\mathrm{cm}^{-1}\right)$ : 3420, 2920, 2627, 1623, 1410, 1276, 1119, 970, 738.

2-(2-Chlorophenyl) benzimidazole [28]: Light pink red solid; m.p: $231^{\circ} \mathrm{C}-233^{\circ} \mathrm{C}$; ${ }^{1} \mathrm{H}$ NMR (DMSO-d ${ }_{6}$ ): $\delta 12.80$ (br s, 1H), $7.91-0.89(\mathrm{~m}, 1 \mathrm{H}), 7.67-7.62(\mathrm{~m}$, 3H), 7.57 - 7.52 (m, 2H), 7.25 - 7.23 (m, 2H); (LC-MS) $\mathrm{m} / \mathrm{z}: 229.04[\mathrm{M}+\mathrm{H}]^{+}$

2-(3-Chlorophenyl) benzimidazole [28]: Colourless solid; m.p: $234^{\circ} \mathrm{C}-236{ }^{\circ} \mathrm{C} ;{ }^{1} \mathrm{H}$ NMR (DMSO-d ${ }_{6}$ ): $\delta 13.06$ (br s, 1H), 8.40 (s, 1H), 8.27 (d, $J=6.8 \mathrm{~Hz}, 1 \mathrm{H}), 7.81$ 7.72 (m, 4H), 7.49 - 7.47 (m, 2H); (LC-MS) m/z: 229.04 $[\mathrm{M}+\mathrm{H}]^{+}$

2-(4-Chlorophenyl) benzimidazole [29]: Colour less solid; m.p: $289^{\circ} \mathrm{C}-291^{\circ} \mathrm{C}$; ${ }^{1} \mathrm{H}$ NMR (DMSO-d ${ }_{6}$ ): $\delta 12.9$ (br s, 1H), 8.15 (d, $J=8 \mathrm{~Hz}, 2 \mathrm{H}), 7.64-7.49$ (m, 4H), 7.20 (d, $J=8 \mathrm{~Hz}, 2 \mathrm{H})$; (LC-MS) m/z: 229.04 [M + H]

2-o-tolylbenzimidazole [27]: Colour less solid; m.p: $220^{\circ} \mathrm{C}-222^{\circ} \mathrm{C} ;{ }^{1} \mathrm{H}$ NMR (DMSO-d ${ }_{6}$ ): $\delta 13.03$ (br s, $1 \mathrm{H}$ ), 7.82 - 7.79 (m, 3H), 7.60 - 7.58 (m, 1H), 7.56 - 7.45 (m, 4H), 2.58 (s, 3H); (LC-MS) m/z: $209.10[\mathrm{M}+\mathrm{H}]^{+}$

2-p-tolylbenzimidazole [27]: Colourless solid; m.p: $265^{\circ} \mathrm{C}-267^{\circ} \mathrm{C}$; ${ }^{1} \mathrm{H}$ NMR (DMSO-d 6 ): $\delta 12.81$ (br s, $1 \mathrm{H}$ ), $8.06(\mathrm{~d}, J=8 \mathrm{~Hz}, 2 \mathrm{H}), 7.56(\mathrm{~m}, 2 \mathrm{H}), 7.36$ (d, $J=8 \mathrm{~Hz}$, 2H), 7.19 (m, 2H), 2.38 (s, 3H); (LC-MS) m/z: 209.10 $[\mathrm{M}+\mathrm{H}]^{+}$

2-(2-Methoxyphenyl) benzimidazole [30]: Colourless solid; m.p: $173^{\circ} \mathrm{C}-175^{\circ} \mathrm{C}$; ${ }^{1} \mathrm{H}$ NMR (DMSO- $\mathrm{d}_{6}$ ): $\delta 13.5$ (br s, 1H), 8.29 (d, J= $7.2 \mathrm{~Hz}, 1 \mathrm{H}), 7.76-7.74$ (m, 2H), 7.63 - 7.59 (m, 1H), 7.39 - 7.32 (m, 3H), 7.22 - 7.18 (m, 1H), 4.06 (s, 3H); (LC-MS) m/z: $225.07[\mathrm{M}+\mathrm{H}]^{+}$

2-(4-Methoxyphenyl) benzimidazole [27]: Colourless solid; m.p: $218^{\circ} \mathrm{C}-221^{\circ} \mathrm{C}$; ${ }^{1} \mathrm{H}$ NMR (DMSO- $\mathrm{d}_{6}$ ): $\delta 12.90$ (br s, $1 \mathrm{H}), 8.21$ (d, $J=8.4 \mathrm{~Hz}, 2 \mathrm{H}), 7.70-7.68(\mathrm{~m}$, 2H), 7.38 - 7.36 (m, 2H), 7.21 (d, $J=8.8 \mathrm{~Hz}, 2 \mathrm{H}), 3.88$ (s, 3H); (LC-MS) m/z: $225.07[\mathrm{M}+\mathrm{H}]^{+}$

2-(3-nitrophenyl) benzimidazole [29]: Off-white so- 
lid; m.p: $203^{\circ} \mathrm{C}-205^{\circ} \mathrm{C}$; ${ }^{1} \mathrm{H}$ NMR (DMSO-d 6 ): $\delta 13.2$ (br s, $1 \mathrm{H}), 9.02$ (s, 1H), 8.60 (d, $J=7.6 \mathrm{~Hz}, 1 \mathrm{H}), 8.33$ (d, $J=$ $7.9 \mathrm{~Hz}, 1 \mathrm{H}), 7.85$ (t, $J=7.9 \mathrm{~Hz}, 1 \mathrm{H}), 7.7$ - 7.52 (m, 2H), 7.25 (t, $J=6.8 \mathrm{~Hz}, 2 \mathrm{H})$; (LC-MS) m/z: $240.06[\mathrm{M}+\mathrm{H}]^{+}$

2-benzylbenzimidazole [27]: Off white solid; m.p: 177 - $179^{\circ} \mathrm{C} ;{ }^{1} \mathrm{H}$ NMR (DMSO-d ${ }_{6}$ ): $\delta 13.0$ (br s, $\left.1 \mathrm{H}\right), 7.52$ - 7.50 (m, 2H), 7.34 - $7.16(\mathrm{~m}, 7 \mathrm{H}), 4.21$ (s, 2H); (LC-MS) m/z: $209.10[\mathrm{M}+\mathrm{H}]^{+}$

\section{Acknowledgements}

The authors are very much grateful to the management of Chalapathi Institute of Engineering and Technology, Guntur, A.P, India, for providing moral support in carrying out this work.

\section{REFERENCES}

[1] H. Zarrinmayeh, A. M. Nunes, P. L. Ornstein, D. M. Zimmerman, M. B. Arnold, D. A. Schober, S. L. Gackenheimer, R. F. Bruns, P. A. Hipskind, T. C. Britton, B. E. Cantrell and D. R. Gehlert, "Synthesis and Evaluation of a Series of Novel 2-[(4-Chlorophenoxy)methyl]-benzimidazoles as Selective Neuropeptide Y Y1 Receptor Antagonists,” Journal of Medical Chemistry, Vol. 41, No. 15, 1998, pp. 2709-2719. http://dx.doi.org/10.1021/jm9706630

[2] Z. Zhu, B. Lippa, J. C. Drach and L. B. Towersend, "Design, Synthesis, and Biological Evaluation of Tricyclic Nucleosides (Dimensional Probes) as Analogues of Certain Antiviral Polyhalogenated Benzimidazole Ribonucleosides,” Journal of Medical Chemistry, Vol. 43, No. 12, 2000, pp. 2430-2437. http://dx.doi.org/10.1021/jm990290y

[3] H. A. Barker, R. D. Smyth, H. Weissbach, J. I. Toohey, J. N. Ladd and B. E. Volcani, "Isolation and Properties of Crystalline Cobamide Coenzymes Containing Benzimidazole or 5,6-Dimethylbenzimidazole," Journal of Biological Chemistry, Vol. 235, No. 2, 1960, pp. 480-488.

[4] J. C. Sih, W. B. Im, A. Robert, D. R. Graber and D. P. Blackmann, "Studies on (H+-K+)-ATPase Inhibitors of Gastric acid Secretion. Prodrugs of 2-[(2-Pyridinylmethyl) sulfinyl]benzimidazole Proton-Pump Inhibitors,” Journal of Medical Chemistry, Vol. 34, No. 3, 1991, pp. 10491062. http://dx.doi.org/10.1021/jm00107a026

[5] A. Figge, H. J. Altenbach, D. J. Brauer and P. Tielmann, "Synthesis and Resolution of 2-(2-Diphenylphosphinylnaphthalen-1-yl)-1-isopropyl-1H-benzoimidazole: A New Atropisomeric $\mathrm{P}, \mathrm{N}$-Chelating Ligand for Asymmetric Catalysis,” Tetrahedron: Asymmetry, Vol. 13, No. 2, 2002, pp. 137-144.

http://dx.doi.org/10.1016/S0957-4166(02)00079-4

[6] E. Alcalde, I. Dinares, L. P. Garcia and T. Roca, “An Advantageous Synthesis of 2-Substituted Benzimidazoles Using Polyphosphoric Acid. 2-(Pyridyl)-1H-benzimidazoles, 1-Alkyl-(1H-benzimidazol-2-yl)pyridinium Salts, Their Homologues and Vinylogues," Synthesis, Vol. 1992, No. 4, 1992, pp. 395-398.

http://dx.doi.org/10.1055/s-1992-26121
[7] V. S. Padalkar, V. D. Gupta, K. R. Phatangare, V. S. Patil, P. G. Umape and N. Sekar, "Indion-190 Resin: Efficient, Environmentally Friendly and Reusable Catalyst for Synthesis of Benzimidazoles, Benzoxazoles and Benzothiazoles," Green Chemistry Letters and Reviews, Vol. 5, No. 2, 2012, pp. 139-145. http://dx.doi.org/10.1080/17518253.2011.585666

[8] R. R. Nagawade and D. B. Shinde, "BF 3 . $\mathrm{OEt}_{2}$ Promoted Solvent Free Synthesis of Benzimidazole Derivatives," Chinese Chemical Letters, Vol. 17, No. 4, 2006, pp. 453456.

[9] M. Kidwai, A. Jahan and D. Bhatnagar, "Polyethylene Glycol: A Recyclable Solvent System for the Synthesis of Benzimidazole Derivatives Using CAN as Catalyst," Journal of Chemical Sciences, Vol. 122, No. 4, 2012, pp. 607-612. http://dx.doi.org/10.1007/s12039-010-0095-7

[10] P. Sun and Z. Hu, "The Convenient Synthesis of Benzimidazole Derivatives Catalyzed by $\mathrm{I}_{2}$ in Aqueous Media," Journal of Heterocyclic Chemistry, Vol. 43, No. 3, 2006, pp. 773-775. http://dx.doi.org/10.1002/jhet.5570430338

[11] B. Sadeghi and M. G. Nejad, "Silica Sulfuric Acid: An Eco Friendly and Reusable Catalyst for Synthesis of Benzimidazole Derivatives,” Journal of Chemistry, Vol. 2013, 2013, Article ID: 581465.

[12] R. Trivedi, S. K. De and R. A. Gibbs, "A Convenient One-Pot Synthesis of 2-Substituted Benzimidazoles," Journal of Molecular Catalysis A: Chemical, Vol. 245, No. 1-2, 2006, pp. 8-11. http://dx.doi.org/10.1016/j.molcata.2005.09.025

[13] R. G. Jacob, L. G. Dutra, C. S. Radatz, S. R. Mendes, G. Perin and E. Lenardao, "Synthesis of 1,2-Disubstituted Benzimidazoles Using $\mathrm{SiO}_{2} / \mathrm{ZnCl}_{2}$," Tetrahedron Letters, Vol. 50, No. 13, 2009, pp. 1495-1497.

http://dx.doi.org/10.1016/j.tetlet.2009.01.076

[14] R. K. Kumar, P. V. V. Satyanarayana and S. B. Reddy, "NaHSO${ }_{4}-\mathrm{SiO}_{2}$ Promoted Synthesis of Benzimidazole Derivatives," Archives of Applied Science Research, Vol. 4, No. 3, 2012, pp. 1517-1521.

[15] C. Mukhopadhyay and P. K. Tapaswi, "PEG-Mediated Catalyst-Free Expeditious Synthesis of 2-Substituted Benzimidazoles and Bis-Benzimidazoles under SolventLess Conditions,” Tetrahedron Letters, Vol. 49, No. 43, 2008, pp. 6237-6240. http://dx.doi.org/10.1016/j.tetlet.2008.08.041

[16] K. Bahrami, M. M. Khodaei and F. Naali, " $\mathrm{H}_{2} \mathrm{O}_{2}$ / $\mathrm{Fe}\left(\mathrm{NO}_{3}\right)_{3}$-Promoted Synthesis of 2-Arylbenzimidazoles and 2-Arylbenzothiazoles" Synlett, Vol. 2009, No. 4, 2009, pp. 569-572.

http://dx.doi.org/10.1055/s-0028-1087911

[17] L. Wang, J. Sheng, H. Tian, et al., “An Efficient Procedure for the Synthesis of Benzimidazole Derivatives Using $\mathrm{Yb}(\mathrm{OTf})_{3}$ as Catalyst Under Solvent-Free Conditions," Synthetic Communications, Vol. 34, No. 23, 2004, pp. 4265-4272. http://dx.doi.org/10.1081/SCC-200039340

[18] A. Loupy, A. Petit, J. Hamelin, et al., "New Solvent-Free Organic Synthesis Using Focused Microwaves," Synthesis, Vol. 1998, No. 9, 1998, pp. 1213-1234. http://dx.doi.org/10.1055/s-1998-6083

[19] G. V. Reddy, V. V. V. N. S. Ramarao, B. Narsaiah, et al., 
"A Simple and Efficient Method for the Synthesis of Novel Trifluoromethyl Benzimidazoles under Microwave Irradiation Conditions," Synthetic Communications, Vol. 32, No. 16, 2002, pp. 2467-2476. http://dx.doi.org/10.1081/SCC-120003394

[20] G. Penieres, I. Bonifas, G. Lopez, et al., "Synthesis of Benzimidazoles in Dry Medium," Synthetic Communications, Vol. 30, No. 12, 2000, pp. 2191-2195. http://dx.doi.org/10.1080/00397910008087397

[21] K. Bougrin, A. Loupy, A. Petit, et al., "Nouvelle Voie de Synthèse des 2-Trifluorométhylarylimidazoles sur Montmorillonite K10 en 'Milieu Sec' Sous Micro-Onde," Tetrahedron. Vol. 57, No. 1, 2001, pp. 163-168. http://dx.doi.org/10.1016/S0040-4020(00)00992-3

[22] S. R. Kumar, Y. Venkateswarlu and P. Leelavathi, "Synthesis of 1,5-Benzodiazepines Catalyzed by Zinc Triflate in Solvent-Free Medium," Asian Journal of Chemistry, Vol. 23, No. 4, 2011, pp. 1611-1614.

[23] X. Zhu and A. Ganesan, "Regioselective Synthesis of 3-Alkylindoles Mediated by Zinc Triflate,” The Journal of Organic Chemistry, Vol. 67, No. 8, 2002, pp. 2705-2708. http://dx.doi.org/10.1021/jo010996b

[24] K. C. Lekhok, D. Bhuyan, D. Prajapati and R. C. Boruah, "Zinc Triflate: A Highly Efficient Reusable Catalyst in the Synthesis of Functionalized Quinolines via Friedlander Annulations," Molecular Diversity, Vol. 14, No. 4, 2010, pp. 841-846. http://dx.doi.org/10.1007/s11030-009-9214-0

[25] V. N. Kumar, G. Sridhar, E. Laxminarayana, B. Mallaiah and T. Giri, "Selective Deprotection of $t$-Butyldimethylsilyl
(TBDMS) Ethers Using Zinc (II) Trifluoromethanesulfonate,” Journal of Drug Chemistry (IJPI), Vol. 2, No. 10, 2012, 4p.

[26] D. Atukuri, R. K. Ravindra and P. K. Pramod, “Zinc Triflate Catalyzed Facile Synthesis of Novel 1,2,4-Trizolinone Derivatives Using 3-Arylsydnone as Synthons," Letters in Organic Chemistry, Vol. 10, No. 7, 2013, pp. 510-517. http://dx.doi.org/10.2174/1570178611310070009

[27] A. J. Blacker, M. M. Farah, M. I. Hall, S. P. Marsden, O. Saidi and J. M. J. Williams, "Synthesis of Benzazoles by Hydrogen-Transfer Catalysis,” Organic Letters, Vol. 11, No. 9, 2009, pp. 2039-2042. http://dx.doi.org/10.1021/ol900557u

[28] S. B. Sapakal, K. F. Shelke, S. S. Sonar, B. B. Shingate and M. S. Shingare, "Acidic Ionic Liquid Catalyzed Environmentally Friendly Synthesis of Benzimidazole Derivatives," Bulletin of the Catalysis Society of India, Vol. 2, 2009, p. 78.

[29] V. A. Sontakke, S. Ghosh, P. P. Lawande, B. A. Choapade and V. S. Shinde, "A Simple, Efficient Synthesis of 2-Aryl Benzimidazoles Using Silica Supported Periodic Acid Catalyst and Evaluation of Anticancer Activity," ISRN Organic Chemistry, Vol. 2013, 2013, Article ID: 453682.

[30] J. Peng, M. Ye, C. Zong, F. Hu, L. Feng, X. Wang and C. Chen, "Copper-Catalyzed Intramolecular C-N Bond Formation: A Straightforward Synthesis of Benzimidazole Derivatives in Water," The Journal of Organic Chemistry, Vol. 76, No. 2, pp. 716-719. http://dx.doi.org/10.1021/jo1021426 